\title{
Xuesaitong Soft Capsule (Chinese Patent Medicine) for the Treatment of Unstable Angina Pectoris: A Meta-Analysis and Systematic Review
}

\author{
Xiaochen Yang, ${ }^{1}$ Xingjiang Xiong, ${ }^{1}$ Heran Wang, ${ }^{2}$ Guoyan Yang, ${ }^{3}$ and Jie Wang ${ }^{1}$ \\ ${ }^{1}$ Department of Cardiology, Guang'anmen Hospital, China Academy of Chinese Medical Sciences, Beixiange 5, Xicheng District, \\ Beijing 100053, China \\ ${ }^{2}$ Cancer Research Institute, Central South University, Changsha, Hunan 410078, China \\ ${ }^{3}$ Centre for Evidence-Based Chinese Medicine, Beijing University of Chinese Medicine, Beijing 100029, China
}

Correspondence should be addressed to Jie Wang; wangjie_gamyy@126.com

Received 10 November 2013; Accepted 19 December 2013

Academic Editor: Bo Feng

Copyright (C) 2013 Xiaochen Yang et al. This is an open access article distributed under the Creative Commons Attribution License, which permits unrestricted use, distribution, and reproduction in any medium, provided the original work is properly cited.

\begin{abstract}
Objective. To provide a systematic review to evaluate the effectiveness and safety of Xuesaitong soft capsule (XST) in treating unstable angina (UA). Methods. An extensive search of 6 medical databases was performed up to August 2013. Randomized controlled trials (RCTs) involving XST alone or combined with conventional drugs versus conventional drugs were included. A meta-analysis of reduction of angina symptoms and electrocardiogram (ECG) improvement was performed to evaluate the effects of XST on UA. Results. After researching, a total of 6 RCTs with 716 participants were included. Our review showed that XST combined with conventional drugs had significant effect on relieving angina symptoms (RR: $1.14[1.07,1.22] ; P=0.0001$ ) and improving ECG (RR: $1.26[1.12,1.42] ; P=0.0001)$ compared with conventional drugs alone. Conclusions. XST appears to have beneficial effects on improvement of ECG, reduction of angina symptoms, and decreasing the frequency and duration of angina attack in participants with UA. However, the findings should be interpreted with caution due to the poor methodological quality of the included trials.
\end{abstract}

\section{Introduction}

Coronary artery disease (CHD) is one of the leading causes of death in most developed and some developing countries [1, 2]. Antiplatelet agents and anticoagulants have demonstrated variable clinical effects in coronary heart disease (CHD), including unstable angina pectoris (UA) and acute myocardial infarction (AMI) [3, 4]. Among the antiplatelet agents, aspirin has been shown to reduce the risk for thrombosis and ischemic events. However, the possibility of aspirin resistance, which has been described as a number of phenomena, including antithrombotic complications, prolongation of the bleeding time, and inhibition of thromboxane biosynthesis, provides an impetus for researching new medicine with high effectiveness and fewer adverse effects [5].

Sanqi, also known as radix notoginseng, is a hemostatic herbal medicine that may have protective effect in patients with UA [6] and has been used for cardiovascular diseases for hundreds of years in China. Recently, with the growing and sustained interest in the benefits of Chinese herbal medicine $(\mathrm{CHM})$ and potential drug interactions with western medications, Sanqi called much attention for its good cardiovascular effects, including inhibition of platelet aggregation, increasing blood flow, improving left ventricular diastolic function in hypertensive patients, and anti-inflammatory effect [7, 8]. Radix notoginseng consists of complex compounds, including saponin, dencichine, polysaccharides, amino acids, flavonoids, phytosterols, fatty acids, volatile oils, aliphatic acetylene hydrocarbons, and trace elements. Panax notoginseng saponins (PNS) are one of the main active ingredients of Radix notoginseng. Until now, twenty-seven saponins were identified and nine of them were notoginsenoside R1, ginsenoside Rb1, Rb2, Rb3, Rc, Rd, Re, Rf, and Rg1 $[9,10]$. Most of these monomer components are 20(S)-protopanaxadiol and 20(S)-protopanaxatriol . Xuesaitong soft capsule (Kunming Samflaming Pharmacy Group Co., Ltd., Kunming, China) 
mainly consists of PNS. The total dose is 100-200 mg for one day. Modern researches have shown that Xuesaitong soft capsule (XST) can stanch bleeding, invigorating and supplementing blood for treating UA $[11,12]$.

Several lines of evidence published in China have reported that XST can enhance the effect of relieving of angina symptoms, decreasing the dosage of nitroglycerin, and lessening adverse effects for patients with UA [19-22]. However, the lines of evidence supporting or disproving these cardiovascular effects have not been well systematically reviewed. In this paper, we evaluated the effect of XST through a rigorous systematic review and meta-analysis of randomized trials.

\section{Methods}

2.1. Study Selection and Search Strategy. The following electronic databases including PubMed, the Cochrane Center Controlled Trials Register (2013), EMBASE (19802013), Chinese National Knowledge Infrastructure (CNKI, 1979-August 2013), Chinese Biomedical Literature Database (CBM, 1978-August 2013), Chinese Scientific Journal Database (VIP, 1989-August 2013) were searched by two authors (X. Yang and H. Wang). The titles and abstracts of potentially relevant studies were identified through searching and reviewed independently by 3 reviewers (G. Yang, H. Wang, and X. Xiong). We selected randomized controlled trials that evaluate the cardiovascular effects of XST for UA. The English searching terms were used individually or combined including "unstable angina pectoris," "Xuesaitong soft capsule," "randomized controlled trial," "controlled clinical trial," "randomly," "trial," "randomised" and "randomized." The Chinese searching terms were used individually or combined including those for the generic name of unstable angina pectoris ("Bu_wen_ding_xing_xian_jiao_tong”), Xuesaitong soft capsule ("Xue_sai_tong_ruan_jiao_nang"), and randomized ("sui_ji"). For discrepancies in the process of selection, whether to include or exclude a study was resolved by consensus with other investigator (X. Yang).

2.2. Types of Interventions and Participants. The included trials were designed to compare the effectiveness and safety of XST used alone or in combination with conventional drugs versus conventional drugs alone. The intervention group was treated with XST alone or combined with conventional drugs for patients with UA regardless of manufactures, dose, and duration. The participants who are suffering from and have been diagnosed with UA should be included regardless of the severity. The diagnosis of UA $[23,24]$ was according to "ACCF/AHA Guideline for the Diagnosis and Management of Patients with Unstable Ischemic Heart Disease (ACCF/AHA)" or "the International Society and Federation of Cardiology/World Health Organization (ISFC/WHO)".

2.3. Types of Outcome Measures. According to the severity and treatment of diseases, the primary outcome measures were mortality due to ischemic heart disease or incidence of heart events (e.g., AMI, severe arrhythmia, heart failure, and revascularization). The secondary outcome measures were: (1) reduction of angina symptoms (e.g., effective symptomatic improvements should achieve at least $50 \%$ or $80 \%$ reduction in frequency of feeling of angina chest pain [24, 25]), (2) Electrocardiogram (ECG) improvement (e.g., effective ECG improvements should achieve at least $0.05 \mathrm{mV}$ lowering at ST segment in ECG or nearly normal ECG during an exercise test $[24,25])$, (3) frequency of angina attack, and (4) duration of angina attack. The followup and adverse events were also measured.

2.4. Data Extraction and Quality Assessment. The qualities of included RCTs were assessed according to several specific domains including random sequence generation, allocation concealment, blinding of participants and personnel, blinding of outcome assessment, incomplete outcome data, and selective reporting. Two authors ( $\mathrm{H}$. Wang, G. Yang) independently assessed the methodological quality of included RCTs according to Cochrane Handbook: such information was provided: (a) a description of included study and (b) their judgment on the risk of bias as a consequence of this. This judgment is categorized by using one of the three answers: "high risk," "unclear risk," and "low risk". About the included RCTs, the following data were extracted: authors of study, year of publication, sample size and age of participants, detailed information of interventions and controls, outcome measures, and adverse events.

2.5. Statistical Analysis. Meta-analyses of RCTs were performed by using RevMan 5.1 software. We summarized data using risk ratios (RR) with 95\% confidence intervals (CI) for dichotomous outcomes or mean difference (MD) with 95\% CI for continuous outcomes. We assessed heterogeneity using both the Chi-squared test and the $I$-squared statistic. If $I$-squared value was greater than $25 \%$, we pooled data using a fixed-effect model; otherwise we use random effects model [26].

\section{Result}

3.1. Description of Studies. A flow chart (Figure 1) showed the search process and study selection. We included 6 RCTs [13-18] for this systematic review. All RCTs were conducted in China and published in full. The detailed characteristics of the included trials were listed in Table 1. The 6 RCTs involved 716 participants with UA, aged between 37 and 77 . The duration of treatment varied from 4 weeks to 8 weeks, with an average duration of 4.6 weeks. The dosage of XST was two capsules twice a day. There was one comparison: XST plus conventional drugs versus conventional drugs. Reductions in angina symptoms and improvement in ECG were the most commonly measured outcomes in the included studies. One trial [15] adopted mortality as primary outcome.

3.2. Risk of Bias Assessment. The included studies indicated randomization with a single-center, parallel design, but most of them did not describe it clearly. Only one trial [13] reported that the random sequence was generated by random number 


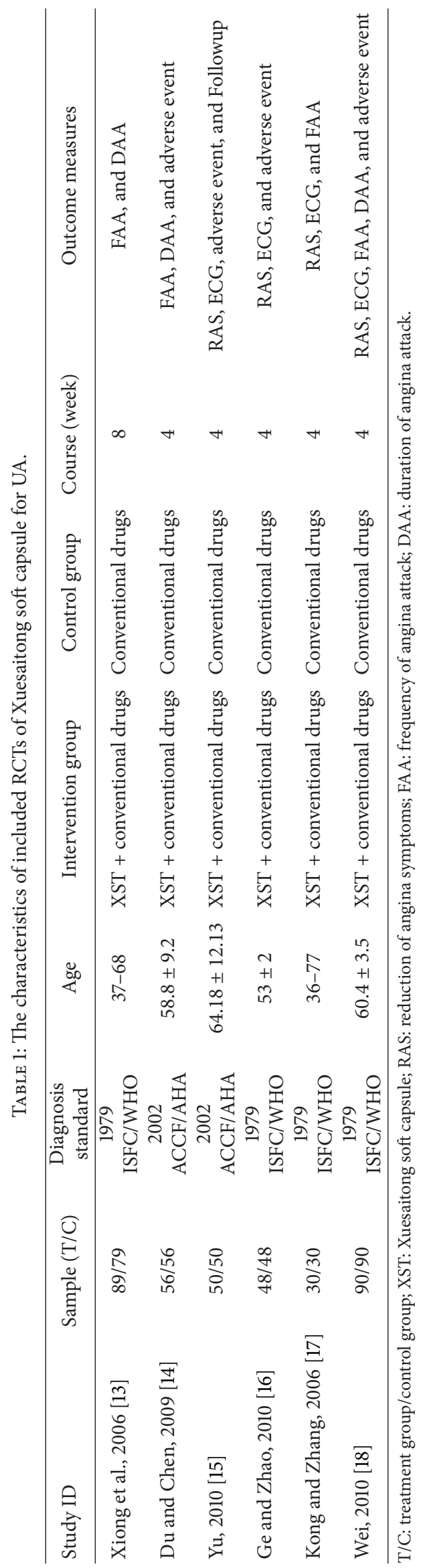




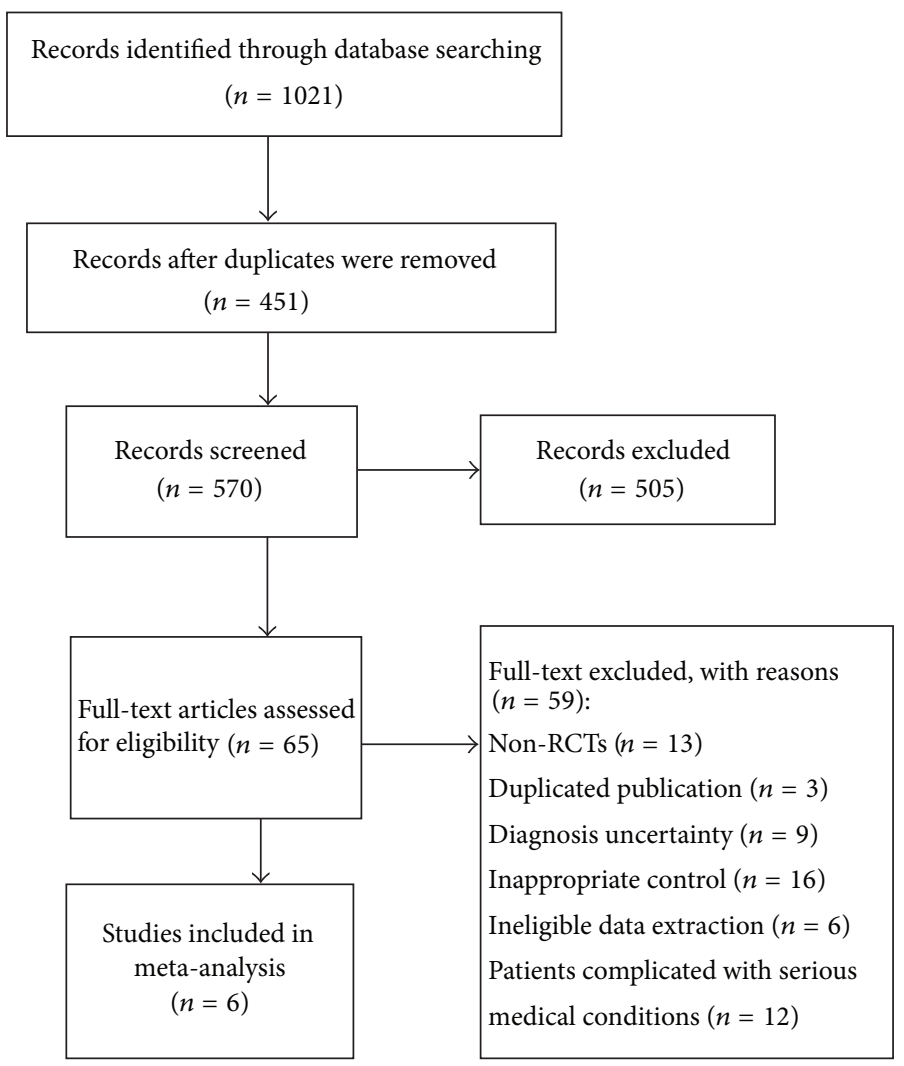

FIGURE 1: Flow chart of study search and selection.

table. None of the trials describe allocation concealment and methods of assessing compliance. One trial [15] reported dropout or withdrawal, but none of the trials had a pretrial estimation of sample size, which indicated the lack of statistical power to ensure appropriate estimation of the therapeutic effect.

\subsection{Effects of Interventions}

3.3.1. Primary Outcomes. After four months of followup, one trial [15] reported 1 case of severe arrhythmia in intervention group and 2 cases of AMI in control group.

\subsubsection{Secondary Outcomes}

(i) Reduction of Angina Symptoms (RAS). Compared with conventional medicine, four individual trials [15-18] with 218 patients reporting reduction of angina symptoms after treatment favored XST plus conventional medicine. Homogeneity in the results is showed $\left(P=0.86, I^{2}=0 \%\right)$. Thus, we did a quantitative data synthesis (meta-analysis) by fixedeffects model. The outcome shows a statistically significant difference in favor of the combination group of XST and conventional drugs (RR: $1.14[1.07,1.22] ; P=0.0001)$. It is suggested that XST plus conventional drugs had a better effect on relieving symptoms of angina (Figure 2).

(ii) ECG Improvement. Compared with conventional medicine, four individual trials [15-18] with 218 patients reporting
ECG improvement after treatment favored XST plus conventional medicine. Homogeneity in the results is showed $(P=$ $\left.0.74, I^{2}=0 \%\right)$. Thus, we did a quantitative data synthesis (meta-analysis) by fixed-effects model. The outcome shows a statistically significant difference in favor of the combination group of XST and conventional drugs (RR: 1.26 [1.12, 1.42]; $P=0.0001)$. It is suggested that XST plus conventional drugs had a better effect on improving ECG (Figure 3).

(iii) Frequency of Angina Attack. Compared with conventional medicine, four individual trials $[13,14,17,18]$ with 265 patients reporting frequency of angina attack after treatment favored XST plus conventional medicine. No homogeneity in the results is showed $\left(P<0.00001, I^{2}=98 \%\right)$. Thus, we did not adopt a meta-analysis. Xiong et al. [13] indicated that frequency of angina attack which decreased from $3.1 \pm 1.1$ times/day to $0.4 \pm 0.5$ times/day after treatment favored XST plus conventional medicine. The effectiveness was nearly equal to Kong and Zhang [17] which decreased from $6.72 \pm 2.24$ times/day to $0.75 \pm 0.79$ times/day. Another two trials $[15,18]$ also indicated after treatment favored XST plus conventional medicine: frequency of angina attack decreased from $7.82 \pm 1.24$ times/day to $2.86 \pm 0.72$ times/day and from $13.31 \pm 0.79$ times/day to $4.27 \pm 0.87$ times/day, respectively.

(iv) Duration of Angina Attack. Compared with conventional medicine, three individual trials $[13,14,18]$ with 235 patients reporting duration of angina attack after treatment 


\begin{tabular}{|c|c|c|c|c|c|c|c|c|}
\hline \multirow{2}{*}{ Study or subgroup } & \multicolumn{2}{|c|}{ Experimental } & \multicolumn{2}{|c|}{ Control } & \multicolumn{3}{|c|}{ Risk ratio } & \multirow{2}{*}{$\begin{array}{c}\text { Risk ratio } \\
\text { M-H, fixed, 95\% CI }\end{array}$} \\
\hline & Events & Total & Events & Total & Weight & \multicolumn{2}{|l|}{$\mathrm{M}-\mathrm{H}$, fixed, 95\% CI } & \\
\hline Ge and Zhao 2010 & 44 & 48 & 36 & 48 & $18.3 \%$ & \multicolumn{2}{|l|}{$1.22[1.02,1.47]$} & - \\
\hline Kong and Zhang 2006 & 49 & 52 & 43 & 52 & $21.8 \%$ & \multicolumn{2}{|l|}{$1.14[0.99,1.31]$} & \\
\hline Wei 2010 & 84 & 90 & 75 & 90 & $38.1 \%$ & \multicolumn{2}{|l|}{$1.12[1.01,1.25]$} & - \\
\hline Yu 2010 & 48 & 50 & 43 & 50 & $21.8 \%$ & \multicolumn{2}{|l|}{$1.12[0.98,1.27]$} & - \\
\hline \multicolumn{2}{|l|}{ Total $(95 \% \mathrm{CI})$} & 240 & & 240 & $100.0 \%$ & \multicolumn{2}{|l|}{$1.14[1.07,1.22]$} & \\
\hline Total events & 225 & & 197 & & & 0.5 & 0.7 & 1.5 \\
\hline \multicolumn{5}{|c|}{ Heterogeneity: $\chi^{2}=0.78, \mathrm{df}=3(P=0.86) ; I^{2}=0 \%$} & & \multicolumn{2}{|c|}{ Favors control } & Favors experimental \\
\hline \multicolumn{5}{|c|}{ Test for overall effect: $Z=3.87(P=0.0001)$} & & & & \\
\hline
\end{tabular}

FIGURE 2: Forest plot of comparison: XST plus conventional drugs versus conventional drugs, outcome: RAS.

\begin{tabular}{|c|c|c|c|c|c|c|c|c|}
\hline \multirow{2}{*}{ Study or subgroup } & \multicolumn{2}{|c|}{ Experimental } & \multicolumn{2}{|c|}{ Control } & \multicolumn{3}{|c|}{ Risk ratio } & \multirow{2}{*}{$\begin{array}{c}\text { Risk ratio } \\
\text { M-H, fixed, 95\% CI }\end{array}$} \\
\hline & Events & Total & Events & Total & Weight & \multicolumn{2}{|l|}{ M-H, fixed, 95\% CI } & \\
\hline Ge and Zhao 2010 & 42 & 48 & 36 & 48 & $24.8 \%$ & \multicolumn{2}{|l|}{$1.07[0.96,1.42]$} & $\longrightarrow$ \\
\hline Kong and Zhang 2006 & 38 & 52 & 29 & 52 & $20.0 \%$ & \multicolumn{2}{|l|}{$1.31[0.98,1.76]$} & \multirow{2}{*}{$\rightarrow-$} \\
\hline Wei 2010 & 75 & 90 & 61 & 90 & $42.1 \%$ & \multicolumn{2}{|l|}{$1.23[1.04,1.46]$} & \\
\hline Yu 2010 & 28 & 50 & 19 & 50 & $13.1 \%$ & \multicolumn{2}{|l|}{$1.47[0.96,2.27]$} & \\
\hline Total $(95 \% \mathrm{CI})$ & & 240 & & 240 & $100.0 \%$ & $1.26[1.12,1.42]$ & & \\
\hline Total events & \multicolumn{2}{|l|}{183} & 145 & & & & $0.5 \quad 0.7$ & $1.5 \quad 2$ \\
\hline \multicolumn{5}{|c|}{ Heterogeneity: $\chi^{2}=1.27, \mathrm{df}=3(P=0.74) ; I^{2}=0 \%$} & & & \multirow[t]{2}{*}{ Favors control } & \multirow[t]{2}{*}{ Favors experimental } \\
\hline \multicolumn{5}{|c|}{ Test for overall effect: $Z=3.80(P=0.0001)$} & & & & \\
\hline
\end{tabular}

FIGURE 3: Forest plot of comparison: XST plus conventional drugs versus conventional drugs, outcome: ECG.

favored XST plus conventional medicine. No homogeneity in the results is showed $\left(P<0.00001, I^{2}=100 \%\right)$. Thus, we did not adopt a meta-analysis. Xiong et al. [13] indicated that duration of angina attack which decreased from $1.6 \pm 0.5 \mathrm{~min}$ to $0.11 \pm 0.08 \mathrm{~min}$ after treatment favored XST plus conventional medicine. Another two trials $[14,18]$ also indicated after treatment favored XST plus conventional medicine: duration of angina attack decreased from $7.82 \pm$ $1.24 \mathrm{~min}$ to $2.86 \pm 0.72 \mathrm{~min}$ and from $10.23 \pm 1.22 \mathrm{~min}$ to $4.56 \pm 1.08 \mathrm{~min}$ respectively.

3.4. Publication Bias. Due to insufficient number of trials, we failed to perform funnel plot to detect publication bias.

3.5. Adverse Effect. Four of the included trials (66.7\%) [14$16,18]$ reported adverse effects relating to the treatment by XST combined with conventional drugs. The adverse effects only included rash $0.27 \%(1 / 363)$. No severe adverse events were reported.

\section{Discussion}

In this review, we found that compared with conventional drugs, XST demonstrated a potential beneficial effect for
UA on relieving symptoms of angina, improving ECG, and decreasing frequency and duration of angina attack. A total of 6 randomized trials including 716 participants were included. All the RCTs claimed that the positive effect of XST combined with conventional drugs was better than conventional drugs alone. However, we still could not make firm conclusions due to the various durations of treatment and diverse reporting methods in included trials. In addition, the methodological quality of all trials was limited.

The following problems in reporting contribute to the limited methodological quality of most included trials: (1) methods of random sequence generation and allocation concealment: only one trial [13] reported that the random sequence was generated by random number table; most trails were not reported; (2) blinding: blinding of participants and personnel and blinding of outcome assessment were unclear; (3) withdrawal/dropout: one trial [15] reported dropout or withdrawal, but none of the trials had a pretrial estimation of sample size; the detailed reasons of withdrawal/dropout were not reported; (4) information on registration was unclear. We highly recommend further RCTs that should be reported according to the CONSORT Statement [27].

Our systematic review included two major criterions: "ACCF/AHA Guideline for the Diagnosis and Management of Patients with Unstable Ischemic Heart Disease 
(ACCF/AHA)" and "the International Society and Federation of Cardiology/World Health Organization (ISFC/WHO)," to diagnose UA patients. According to the severity and treatment of diseases, reduction of angina symptoms, ECG improvement, frequency of angina attack, duration of angina attack were used as scales to assess the effectiveness of XST for UA. Four trials [15-18] used reduction of angina symptoms and ECG improvement as outcome measurements. Four [13, $14,17,18]$ used frequency of angina attack and three $[13,14,18]$ used duration of angina attack as outcome measurements. The main findings of this systematic review were that XST combined with conventional drugs demonstrated potential effect on relieving symptoms of angina (RR: 1.14 [1.07, 1.22]; $P=0.0001)$ and improving ECG (RR: $1.26[1.12,1.42]$; $P=0.0001)$ compared to conventional drugs alone. There were only a few trials that report the original data about the frequency and duration of angina; however a substantial level of heterogeneity of these data was indicated and metaanalysis was not adopt.

None of the included trials reported severe adverse events possibly related to XST, and the adverse effects only included rash $0.27 \%(1 / 363)$. We cannot draw firm conclusions about the safety of XST since four of six trials did report information on safety. The sample size was limited, and none of trials had a pretrial estimation of sample size, which indicated the lack of statistical power to ensure appropriate estimation of the therapeutic effect. The duration of treatment in most trials was 4 weeks or 8 weeks, so the potential beneficial or harmful effect of XST for treatment of UA might only result from symptomatic changes and short treatment duration. The included trials used the same doses of XST (two capsules twice a day). Since most participants with UA disease require lifelong treatment, the long-term safety of the treatment is still an important concern. The further studies should pay attention to the monitoring and reporting of adverse events and long-term safety by designing a longer duration of treatment and a long-term followup. Traditional Chinese medicine (TCM) is a holistic system of medicine and has unique theories of the diagnosis and treatment [2830]. Although XST has been widely used in cardiovascular diseases in China, the efficacy and safety of Chinese patent medicine remain an issue and need evidence to prove it according to the theory of TCM.

\section{Conclusion}

XST in combination with conventional drugs had significant effect on reducing angina symptoms, improving ECG, and decreasing frequency and duration of angina attack with few side effects for patients with UA. However, the findings should be interpreted with caution due to the low quality of included trials. More rigorously designed, large-sample, randomized controlled trials are warranted to support its clinical use in the future.

\section{Conflict of Interests}

All authors declare that they have no conflicts of interests.

\section{Author's Contribution}

Xiaochen Yang and Xingjiang Xiong contributed equally to this paper.

\section{Acknowledgments}

The current work was partially supported by the National Basic Research Program of China (973 Program, no. 2003CB517103) and the National Natural Science Foundation Project of China (no. 90209011).

\section{References}

[1] E. Braunwald and D. A. Morrow, "Unstable angina: is it time for a requiem?" Circulation, vol. 127, pp. 2452-2457, 2013.

[2] K. Thygesen, J. S. Alpert, and H. D. White, "Universal definition of myocardial infarction," Circulation, vol. 116, no. 22, pp. 26342653, 2007.

[3] F. W. Bar, F. W. Verheugt, J. Col et al., "Thrombolysis in patients with unstable angina improves the angiographic but not the clinical outcome: results of UNASEM, a multicenter, randomized, placebo-controlled, clinical trial with anistreplase," Circulation, vol. 86, no. 1, pp. 131-137, 1992.

[4] S. D. Wiviott and E. Braunwald, "Unstable angina and non-STsegment elevation myocardial infarction. Part I: initial evaluation and management, and hospital care," Annals of Emergency Medicine, vol. 70, no. 3, pp. 525-532, 2004.

[5] C. Patrono, B. Coller, G. A. FitzGerald, J. Hirsh, and G. Roth, "Platelet-active drugs: the relationships among dose, effectiveness, and side effects," Chest, vol. 126, no. 3, supplement, pp. 235S-264S, 2004.

[6] A.-J. Lau, D.-F. Toh, T.-K. Chua, Y.-K. Pang, S.-O. Woo, and H.-L. Koh, "Antiplatelet and anticoagulant effects of Panax notoginseng: comparison of raw and steamed Panax notoginseng with Panax ginseng and Panax quinquefolium," Journal of Ethnopharmacology, vol. 125, no. 3, pp. 380-386, 2009.

[7] C. Pan, Y. Huo, X. An et al., "Panax notoginseng and its components decreased hypertension via stimulation of endothelialdependent vessel dilatation," Vascular Pharmacology, vol. 56, no. 3-4, pp. 150-158, 2012.

[8] G. Liu, B. Wang, J. Zhang, H. Jiang, and F. Liu, “Total panax notoginsenosides prevent atherosclerosis in apolipoprotein Eknockout mice: role of downregulation of CD40 and MMP-9 expression," Journal of Ethnopharmacology, vol. 126, no. 2, pp. 350-354, 2009.

[9] W. Xia, C. Sun, Y. Zhao, and L. Wu, "Hypolipidemic and antioxidant activities of Sanchi (Radix Notoginseng) in rats fed with a high fat diet," Phytomedicine, vol. 18, no. 6, pp. 516-520, 2011.

[10] S. Y. Han, H. X. Li, X. Ma et al., "Evaluation of the antimyocardial ischemia effect of individual and combined extracts of Panax notoginseng and Carthamus tinctorius in rats," Journal of Ethnopharmacology, vol. 145, no. 3, pp. 722-727, 2013.

[11] J.-W. Guo, L.-M. Li, G.-Q. Qiu et al., "Effects of Panax notoginseng saponins on ACE2 and TNF-alpha in rats with postmyocardial infarction-ventricular remodeling," Zhong yao cai, vol. 33, no. 1, pp. 89-92, 2010.

[12] S. Chen, J. Liu, X. Liu et al., "Panax notoginseng saponins inhibit ischemia-induced apoptosis by activating PI3K/Akt pathway in 
cardiomyocytes," Journal of Ethnopharmacology, vol. 137, no. 1, pp. 263-270, 2011.

[13] P. Xiong, Z. N. Wang, J. M. Li et al., "Clinical observation of Xuesaitong soft capsule for unstable angina and effect of ET and GMP-140 in patients with unstable angina," Chinese Remedies and Clinics, vol. 6, no. 11, pp. 879-881, 2006.

[14] Z. A. Du and G. L. Chen, "Clinical observation of Xuesaitong soft capsule for unstable angina and effect of NO, ET, and CRP in patients with unstable angina," Chinese Journal of Modern Drug Application, vol. 3, no. 4, pp. 140-141, 2009.

[15] D. H. Yu, "Clinical observation and analysis of Xuesaitong soft capsule for unstable angina," Clinical Medicine Applicaiton, vol. 19, no. 1, pp. 38-39, 2010.

[16] L. Ge and S. Z. Zhao, "Clinical observation of Xuesaitong soft capsule for unstable angina in 96 cases," Hebei Journal of Traditional Chinese Medicine, vol. 32, no. 8, pp. 1223-1229, 2010.

[17] Y.Z. Kong and H. G. Zhang, "Clinical observation of Xuesaitong soft capsule for unstable angina in 52 cases," Yunnan Journal of Traditional Chinese Medicine, vol. 27, no. 3, pp. 24-25, 2006.

[18] Y. Y. Wei, "Clinical observation of Xuesaitong soft capsule for unstable angina in 90 cases," Chinese Journal of Modern Drug Application, vol. 4, no. 23, pp. 20-24, 2010.

[19] Z. T. Wang and C. Y. Zeng, "Clinical observation of Xuesaitong soft capsule for unstable angina in 103 patients with unstable angina," Chinese Journal of Integrated Traditional and Western Medicine, vol. 24, no. 7, pp. 652-655, 2004.

[20] Q. Y. Wu, "Clinical observation of Xuesaitong soft capsule in combination with nitroglycerin for unstable angina in 42 cases," Guiding Journal of TCM, vol. 15, no. 12, pp. 24-27, 2009.

[21] S. C. Liang, "Clinical observation of Xuesaitong soft capsule for unstable angina in 56 cases," Journal of Practical Traditional Chinese Medicine, vol. 24, no. 9, p. 564, 2008.

[22] X. D. Xiong, "Clinical observation of Xuesaitong soft capsule for unstable angina in 35 cases," Journal of Emergency in Traditional Chinese Medicine, vol. 10, no. 4, pp. 186-189, 2001.

[23] American College of Cardiology and American Heart Association, "ACC/AHA guidelines for the management of patients with unstable angina and non-ST-segment elevation myocardial infarction: executive summary and recommendations," Circulation, vol. 102, pp. 1193-1209, 2000.

[24] E. Rapaport, R. Bernard, and E. Corday, "Nomenclature and criteria for diagnosis of ischemic heart disease. Report of the Joint International Society and Federation of Cardiology/World Health Organization Task Force on standardization of clinical nomenclature," Circulation, vol. 59, no. 3, pp. 607-609, 1979.

[25] Ministry of health of the People's Republic of China, "Clinical research guidelines for new traditional Chinese drug," 1993.

[26] J. P. T. Higgins and S. Green, "Corchrane Reviewers' Handbook 5. 1. 0," in Review Manager (RevMan) [Computer program], Version 5. 1. 0, 2011.

[27] L. Turner, L. Shamseer, D. G. Altman et al., "Consolidated standards of reporting trials (CONSORT) and the completeness of reporting of randomised controlled trials (RCTs) published in medical journals," Cochrane Database of Systematic Reviews, vol. 14, no. 11, 2012.

[28] X. J. Xiong, X. C. Yang, Y. M. Liu, Y. Zhang, P. Q. Wang, and J. Wang, "Chinese herbal formulas for treating hypertension in traditional Chinese medicine: perspective of modern science," Hypertension Research, vol. 36, no. 7, pp. 570-579, 2013.

[29] J. Wang, P. Q. Wang, and X. J. Xiong, "Current situation and reunderstanding of syndrome and formula syndrome in Chinese medicine," Internal Medicine., vol. 2, Article ID 1000113, pp. 1-5, 2012.

[30] J. Wang and X. J. Xiong, "Current situation and perspectives of clinical study in integrative medicine in China," Evidence-Based Complementary and Alternative Medicine, vol. 2012, Article ID 268542, 11 pages, 2012. 


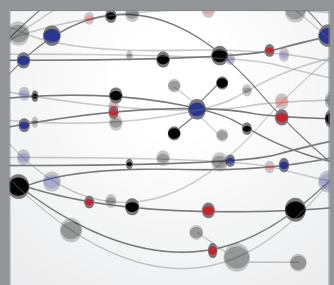

The Scientific World Journal
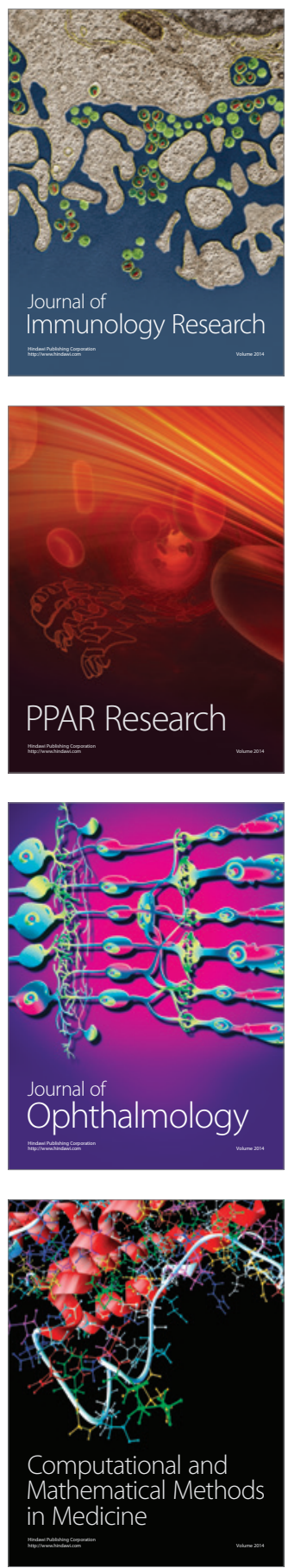

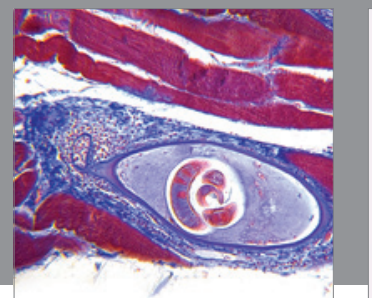

Gastroenterology

Research and Practice
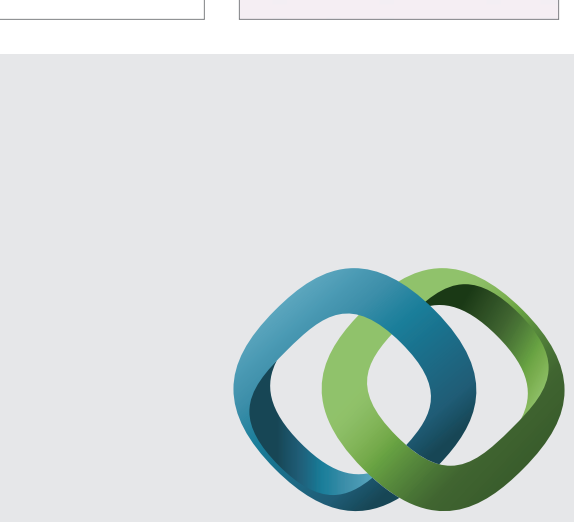

\section{Hindawi}

Submit your manuscripts at

http://www.hindawi.com
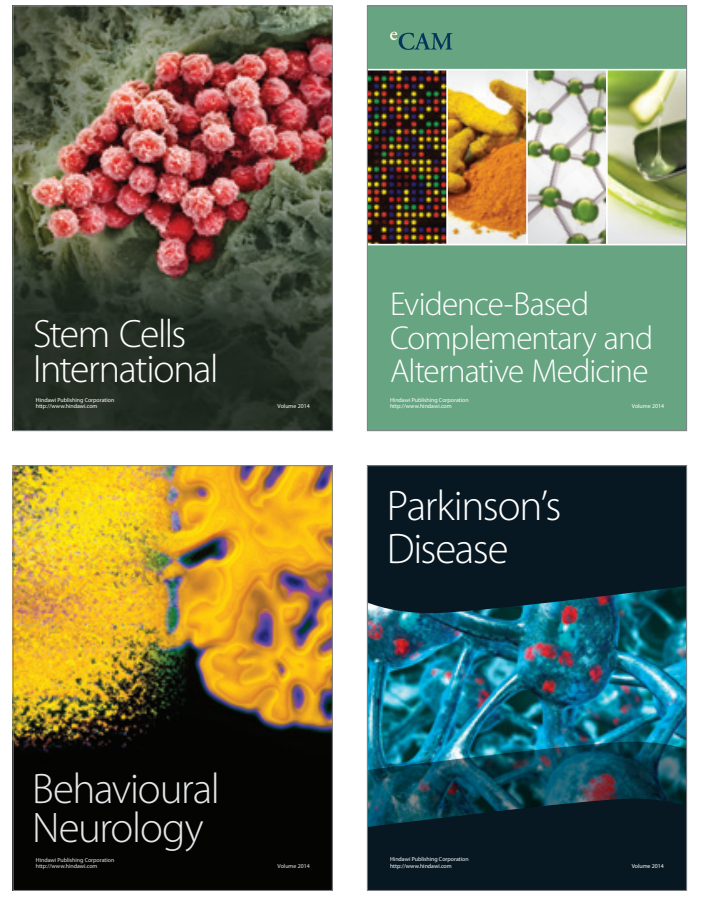
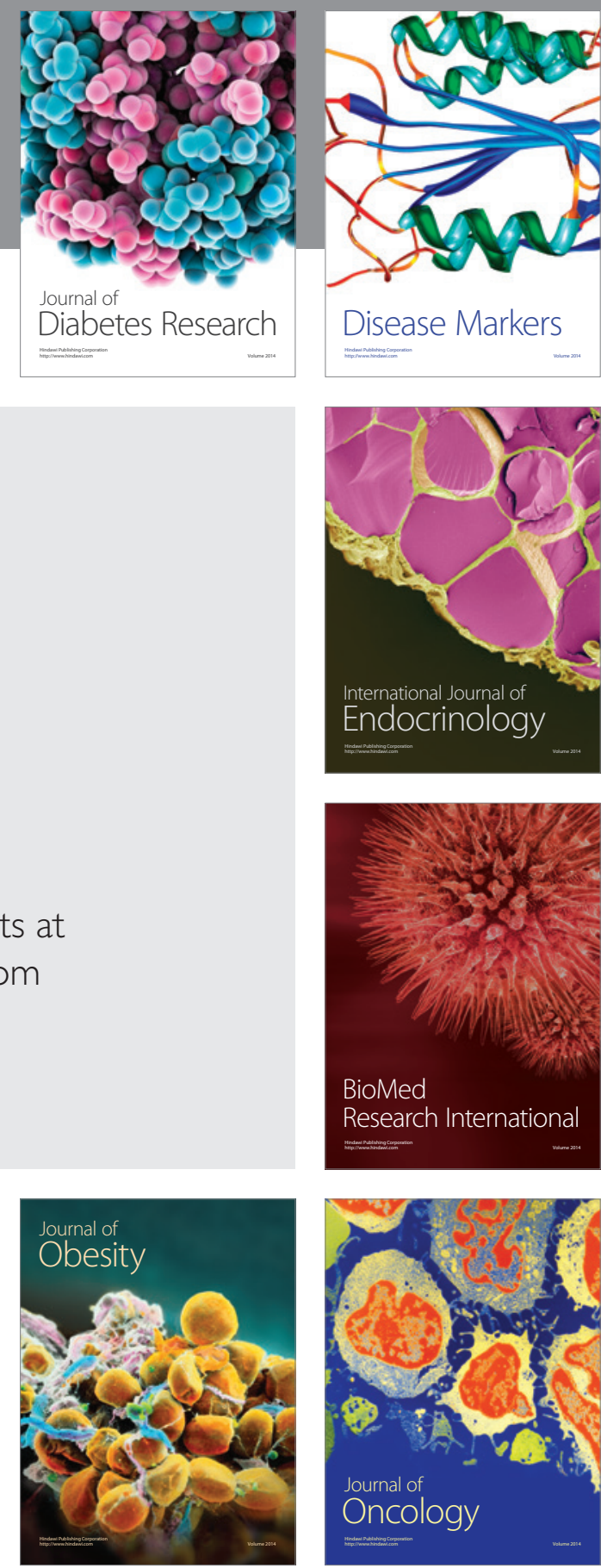

Disease Markers
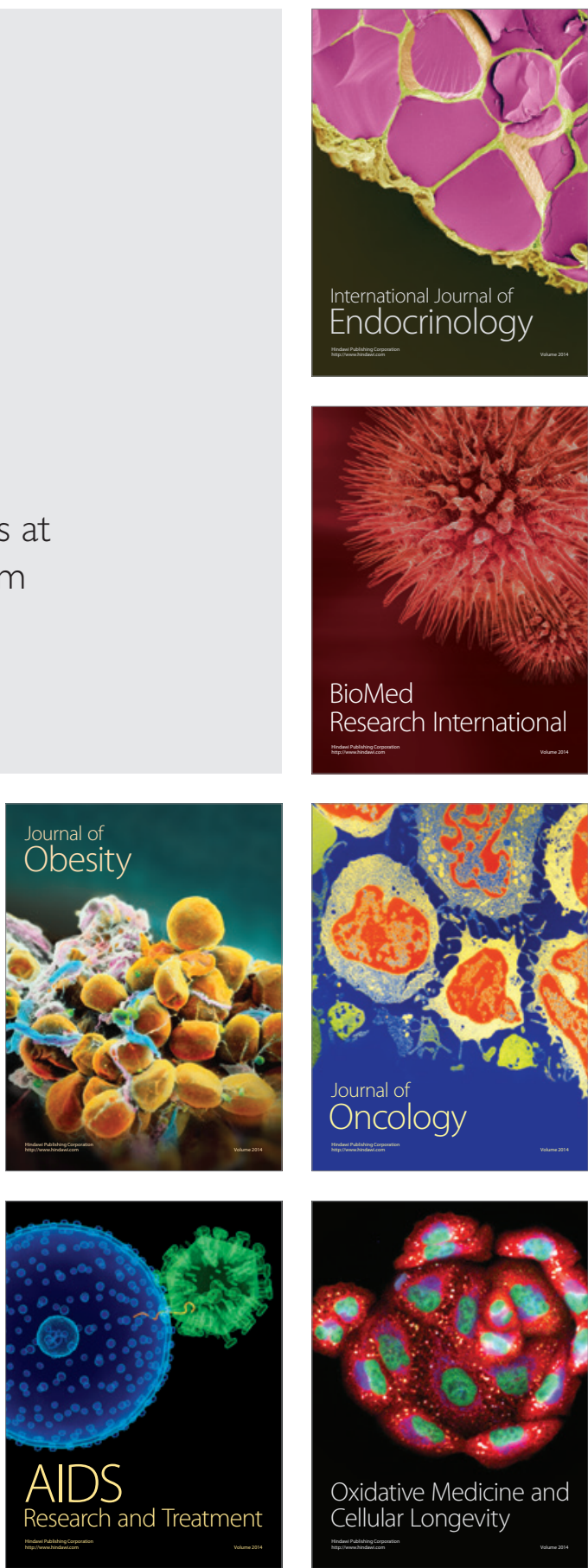